\title{
Spinodal Theory: A Common Rupturing Mechanism in Spinodal Dewetting and Surface Directed Phase Separation (Some Technological Aspects: Spatial Correlations and the Significance of Dipole-Quadrupole Interaction in Spinodal Dewetting)
}

\author{
Satya Pal Singh ${ }^{1,2}$ \\ ${ }^{1}$ Department of Physics and Electronics, Dr. R. M. L. Avadh University, Faizabad, UP 224001, India \\ ${ }^{2}$ Department of Applied Sciences, MMM Engineering College, Gorakhpur, UP 273010, India \\ Correspondence should be addressed to Satya Pal Singh, singh.satyapal@hotmail.com
}

Received 25 July 2010; Revised 11 January 2011; Accepted 11 February 2011

Academic Editor: Nigel Wilding

Copyright (๑) 2011 Satya Pal Singh. This is an open access article distributed under the Creative Commons Attribution License, which permits unrestricted use, distribution, and reproduction in any medium, provided the original work is properly cited.

The emerging structures in spinodal dewetting of thin nano films and spinodal decomposition of binary mixtures are found to be similar with certain differences attributed to the nonlinearities inherent in the wetting forces. This paper deals with the technological aspects of the spinodal processes by giving a brief account of the theory and to correlate the two phenomena termed as spinodal dewetting of thin nanofilms and surface-directed phase separation. The MC simulation micrographs at early stage of spinodal dewetting of a (linear) polymer film confined between two hard walls (using FENE potential between the beads on same chain and Morse potential between inter and intra chain beads) show similarities with surface-directed phase separation (using metropolis algorithm) in creation of holes. The spinodal dewetting is also criticized on the basis of global minimization of free energy emerging from dipole-quadrupole interactions. A novel molecular scale-driving mechanism coming from asymmetric interface formation in spinodal processes is also proposed. It can be believed that the modeling done with the films under confinement of two walls works as a classical mathematical ansatz to the dipole-quadrupole interaction coming from quantum origins and giving rise to lateral interactions in the process reflecting a colossal behavior in thin nano films though weak in nature.

\section{Introduction}

Dewetting of thin films $(<100 \mathrm{~nm})$ have created great interest among physicists and chemists as they see this as new technique to produce nanodevices, for example, polymerbased nanosize organic devices such as nanoscale memory. The emerging structures at early stages in the spinodal dewetting of thin nanofilms in contact with a substrate has been found very much similar to those as in surface directed phase separation (SDPS) [1-4]. The MC simulation patterns in SDPS have shown close resemblance to phase separation followed by dewetting or wetting of one component of the polymer mixtures in contact with a substrate [5]. Thus, SDPS has found importance not only for the study of rupturing or pseudodewetting of spin domains (magnetic memory) but also in the study of the spinodal instabilities leading to dewetting of thin nanofilms or binary mixture of thin films on a surface. Spinodal dewetting of polymer surface by a thin polymer film give rise to patterns of remarkably well-aligned polymer lines with well-defined width and is controlled by the magnitude of the dispersion forces at the interface, which in turn can be varied by changing the thickness of the polymer substrate [6]. Such studies are helpful in understanding the adsorption properties of the (coated) substrates. It has been reported that patterns have shown significant dependence on the interaction of phases with the substrate [7], that is, chemical properties of the substrates.

With the manifold developments in science and technology every year, the sizes of the applied devices are getting more and more compact, and thus the technologists are confronting more and more complications in the production of such devices. The problem comes in controlling the growth processes and in making these devices 
stable. Several groups all over world are dedicated to the synthesis and investigation of functional materials, focusing on the novel size-dependent physics and chemistry that result when atoms and electrons are confined within nanoscale semiconductors and metal clusters. Remarkable variations have been observed in past decades in fundamental electrical, optical, and magnetic properties of functional materials as one progresses from an infinitely extended solid to small domain of material consisting of a countable number of atoms [8]. The cellular targets of versatile quantum-dot beads or polymer microarrays can be changed simply by changing their surface chemistry, and thus the surface must be tailored for different biological applications [9]. It is not easy finding a worthy successor to highly refined microchip technologies $[10,11]$. Molecular-scale electronic devices and its biological counterparts are fast becoming a good bet. A controlled dewetting may be used in the synthesis of nanoscale structures, as G. Reiter at MaxPlanck Institute says that controlled dewetting of magnetized films may be used to make nanoscale memory [12]. The present microchips can be replaced by smarter nanochips in future. The researchers working in the area of dewetting of thin films see this area as a microscope for observing forces working at very small scale. Films formed by gold nanoparticle polyoctylthiophene blends exhibit fluorescence from the circular regions of the morphological structures. The dispersion of the circles can be a signature of the differences in the evaporation/dewetting processes of the two systems during the film formation resulting in the creation of local domains [13].

Wetting or growth has always been a focused area, but at saturation when sizes are getting compact, dewetting has developed a new interest in researchers. The tendency of thin films of liquids and solids to get ruptured or dewetted from surfaces challenges the ingenuity of the engineers and designers. An insulating glaze in a microelectronic chip may allow short-circuit of the film, as it breaks and can tease its manufacturers. Pierre Wiltzius of Lucent Tech. Bell Labs says, "Thin films often are finicky little beasts." Dewetting/selfcleaning of the window glasses in multistory complexes, cars, and other transportation vehicles is always welcomed. Although self-cleaning surface have still got applications in household commodity sector and in biotechnology [14]. Thermal stability of thin polymer films is of importance for various technological applications like coatings and dielectric layers. Study of dewetting can help in understanding the adsorption properties of the (coated) surfaces [15] and a diversity of physical and biological thin film phenomena [4]. A brief introduction of spinodal theory of dewetting and surface directed phase separation process with recent developments in this area along with two (NVT-MC) simulation work for spinodal dewetting of polymer film and for surface directed phase separation, respectively, are presented in this essay. Some new ideas such as the significance of dipolequadrupole interaction in spinodal dewetting giving rise to a completely new route of global minimization free energy, which can alter the dynamics and structure formations in the process, and a novel driving mechanism for molecular scale motors or polymer-based drug transportation from evolution of asymmetric interface formations in spinodal process leading to a potential gradient have been discussed.

\section{The Theory and the Mathematical Modeling}

There are mainly two theories which can explain dewetting of thin films satisfactorily. The first one is the spinodal theory. Spinodal stands for sharp peaks or spines, which are noted when scientists photograph the evolution of the patterns of thin films at different times. The Dutch chemist Anton Vrij hypothesized more than fourty years ago that extremely thin films of liquids (i.e., soap films in air) break its surface by amplifying the amplitude (thermal jiggling). When its amplitude gets the dimension of the thickness of the film, the film is broken [16]. A nearly simultaneous breakup of the initially smooth film takes place at the positions of randomly distributed holes or defects. The average characteristic distance of the initial defects (holes) is proportional to the square of the thickness of the film [17]. The holes then grow, and the rims ahead of the holes eventually merge to form cellular structures. The resulting ribbons are unstable and decay into droplets. The idea of Anton was latter extended to liquid films on both liquid and solid surfaces. The variation in height $h(x, t)$ of the film at different position $x$ and time $t$ are as shown in the Figure 3, which is a simplified and normalized pictorial representation of spines in early stage of dewetting, as the displacements due to capillary action are not taken into account. $h_{0}, q$, and $R$ are, respectively, the initial thickness, the wave vector along $\mathrm{X}$-axis, and the growth rate.

$$
h(x, t)=h_{0}+\operatorname{Exp}(\operatorname{Iqx}) * \operatorname{Exp}(R t)
$$

The vital role is played by van der Waals forces in forming these spines, which arise from displacements of electrons clouds when atoms are pressed together. The van der Waals force is long-range force acting over the span of several hundred of molecules. It can gather the collective muscle to breakdown films of thickness of order ten nanometers [12]. The remarkable and interesting thing is that the van der Waals forces in liquids are very weak in nature in comparison to other intermolecular forces, and surprisingly enough, because of this force, a small thermal vibration or jiggling of any atom or molecule develops as a spinodal wave to dewet a thin film as a consequence. One can raise a question, "why these spines waves are formed?" The answer lies in the fact that the systems have a tendency to be in states of minimum energy and several actions in nature are the manifestations of this principle. If the growth in the amplitude of the disturbance lowers the free energy of the film on substrate, the amplitude grows with time and leads to dewetting, (rupturing of film); otherwise, these spines are damped and the substrate or surface remains wet. Thus, the spreading or vice-versa dewetting depends upon the interactions of the substrate and on any other media if in contact with film [18]. In case of very thin films $<100 \mathrm{~nm}$, the weak van der Waals force gets significance and play vital role in dewetting. Thin Polystyrene films $(<100 \mathrm{~nm})$ on silicon substrates show spinodal dewetting Figure 1 when 


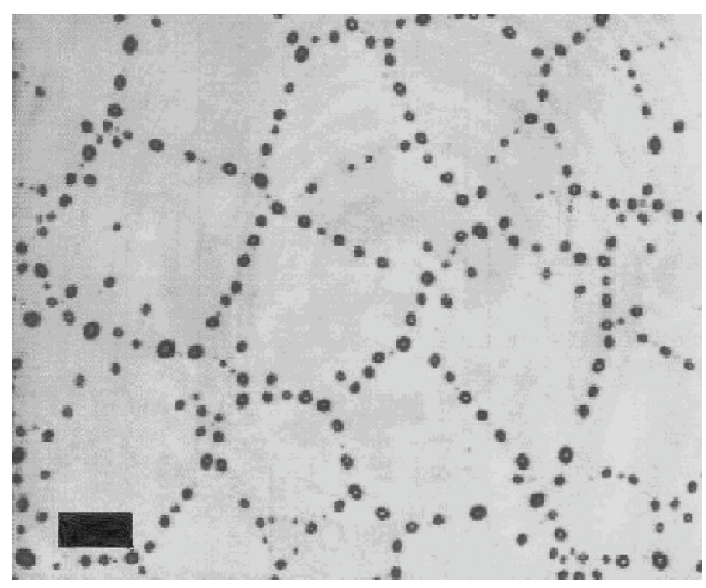

FIGURE 1: Shows micrograph of final polygon pattern after decay of rims into droplets. The initial sample was a $45 \mathrm{~nm}$ thick PS film on wafer A. The length of the bar is $70 \mu \mathrm{m}$. Sharma and Reiter [26].

annealed above their glass-transition temperatures [17] (the glass-transition temperature of very thin film varies with the thickness of the film) $[4,10-16,19,20]$. Buschbaum found the late stage dewetting patterns of thin polystyrene films on silicon oxide substrates to be consistent with the spinodaldewetting theory $[4,14-21]$. Recent atomic force microscopy (AFM) measurements provide good evidence that spinodal process is indeed a mechanism for the dewetting of ultra thin very low molecular weight polystyrene on silicon (liquid on solid dewetting) [4, 9-22].

When liquid films are thick enough but the thickness is less than a few micrometers, spinodal process cannot be responsible for dewetting as the contributions of the excess surface free energy, and the bulk free energy cannot allow it to prevail. Another mechanism may be at work is nucleation. The formation of circular holes originated from large dust particles, defects, or heterogeneities is defined as nucleation. The heterogeneous influences may exist in liquid films, as the interfacial excess surface free energy can withhold the energetically relatively higher energy small regions (drops or nucleus) inside the bulk of the system $[16,23]$. The same is not true for solid films as the interfacial tensions or stresses are not significant enough. Molten solids can have these meta-stable regions in some special cases [24]. The growth of holes leads to the accumulation of the material along the perimeters of the holes. An elevated rim is formed in the process. C. Redon nucleated holes in thin films of alkanes on silicon wafers at temperatures above their glass transition $[4,6-16,19,20,25]$.

A group of physicists at the University of Konstanz have reported a coexisting mechanism of dewetting modes in which surface modulations occurred after the nucleation on a time scale comparable to the growth of the circular holes as the bigger holes developed latter are very nicely circular [16]. The group did its experiments with thin liquid-metal films of $\mathrm{Au}, \mathrm{Cu}$, and $\mathrm{Ni}$ (using Q-switched Laser) on fused silica substrate.

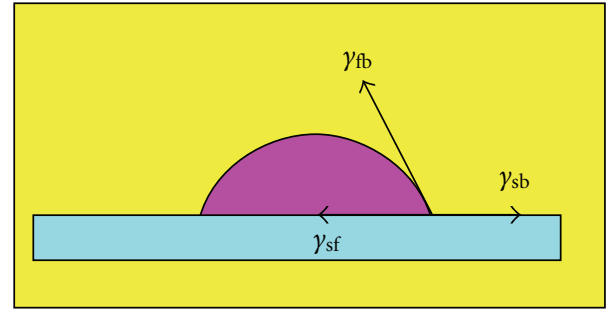

FIgURE 2: Shows interfacial tensions working on a macroscopic drop at a substrate. $\gamma_{\mathrm{fb}}, \gamma_{\mathrm{sf}}$, and $\gamma_{\mathrm{sb}}$ are interfacial tensions between fluid-bounding medium, surface-fluid medium, and surface-bounding medium.

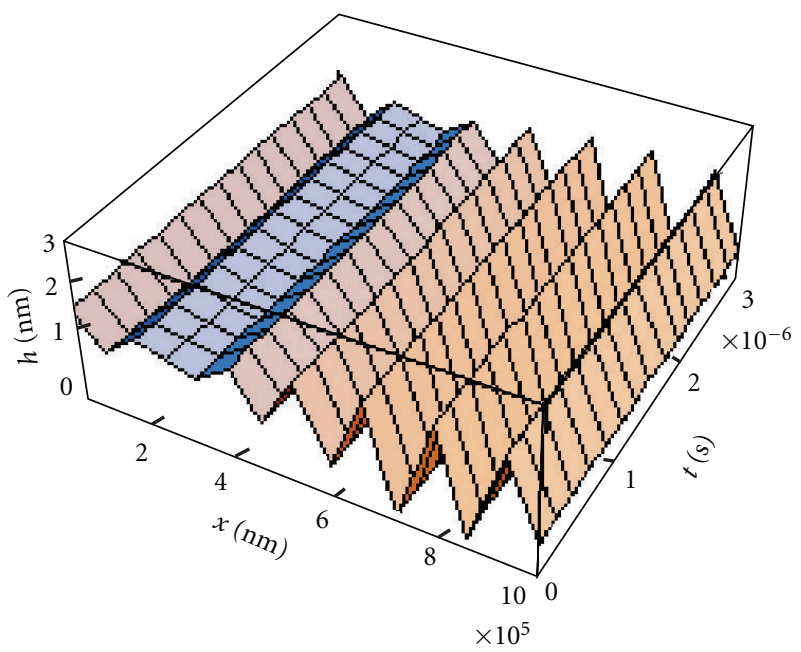

FIGURE 3: A 3D graph representing time evolution of spines at early stages (linear regime) [27].

Thin films subject to long range van der Waals attraction force and extremely short-range repulsion force can undergo a morphological phase separation (MPS). The saturation of initial instability leads to a quasistable periodic structure of nanodrops in stable coexistence with thinner flat films. The equilibrium contact angles of microdrops in MPS are different from the contact angles of corresponding microscopic drops as the significant portion of the microdrops with heights less than about $20 \mathrm{~nm}$ falls in the range in which excess intermolecular interactions are in operation, and thus the bulk region with constant curvature does not exist. Thickness of the surrounding equilibrium films for a microdrop and macrodrop of the same matter are expected to be different. Since the excess energy of the nanodomain depends on its thickness, the equilibrium film pressure and, consequently, the contact angles varies with the size of the microdrops [28]. PS and PS-PEO (a block copolymer) films adsorbed on UV-ozone-treated wafers show morphological phase separation.

Lipson and his coworkers have investigated the breakup of evaporating water film on mica by thinning it from a thickness $500 \mathrm{~nm}$ to a critical thickness $\sim 50 \mathrm{~nm}$ at which the film dewetted the substrate by the formation of growing 
holes $[4,10-25,28-30]$. Water films on other substrates (i.e., glass) show the same behavior. A film of volatile medium in contact with unsaturated vapor is bound to a plane substrate by both van der Waals and polar force [30]. The exchange of atoms or molecules between the two phases, the liquid film, and the vapor phase accounts for the equilibrium thickness. The observed length and time scale are not consistent with the assumption that the rupture is caused by the van der Waals forces [29]. The hydrophobic interaction is said to be at work. The increased strength and range of the hydrophobic attraction may force the onset of instability at higher thickness. The formation of large clusters of solvent cages (i.e., less order or less organization) results in a net increase in the entropy of the system [31]. The acyclic saturated hydrocarbons become more hydrophobic as the carbon chain length increases. So, one may see a possible engineered dewetting via this mechanism.

For technological applications, a controlled dewetting is required. Physicists have reported factors which may be used for the objective. The dynamics of a coexisting molecularly thin and macroscopically thick layers can be controlled by changing the pressure of the vapor phase [30]. Thin films on a nonwettable surface can be stabilized by adding polymer (connectors), which are anchored on the surface and can penetrate into the film [32]. The geometric roughness of a solid substrate can induce wetting [33].

Herminghaus has shown that coupling of various shortrange forces with long-range van der Waals can give rise to different patterns [1-25, 28-34]. Physicists have tried to understand such interactions. A group of researchers report a resonant coupling between the lateral phase separation process of ultra thin polymer blend films $(L<200 \mathrm{~nm})$ and the surface deformation modes [35]. The displacements of the polymer/polymer interface are much larger than the fluctuations at the free surface in the dewetting of PS (polystyrene) by a thin (polymethyl methacrylate) (PMMA) film as the polymer/polymer interfacial tensions are typically an order of magnitude smaller than the polymer surface tension, and thus the interfacial tension dominates in the early stage of spinodal dewetting [22]. Kinetically stable structures are observed in the study of dewetting phenomenon for binary polymer mixtures (nonlinears systems). The steady state itself being unstable, the system dwells on it for anomalously large time [36]. Hopping in adsorbed monolayers can give rise to a dewetting process in monolayer thick film on solid substrates.

\subsection{The Free Energy and the Dynamics of Spinodal Dewetting}

2.1.1. The Microscopic and the Macroscopic Parameters. The wettability of a macroscopic drop of film fluid in presence of bounding fluid is related to the total spreading coefficient $S$ and the equilibrium contact angle $\theta$ of the drop by the Young-Dupre equation

$$
\begin{gathered}
\gamma_{\mathrm{fb}} \cos \theta+\gamma_{\mathrm{sf}}=\gamma_{\mathrm{sb}}, \\
S=\gamma_{\mathrm{sb}}-\gamma_{\mathrm{sf}}-\gamma_{\mathrm{fb}}=\gamma_{\mathrm{fb}} \cos \theta-\gamma_{\mathrm{fb}}
\end{gathered}
$$

where $\gamma_{\mathrm{sb}}$ is the total interfacial tension between the substrate and the bounding fluid, $\gamma_{\mathrm{sf}}$ is the interfacial tension between the substrate and the film fluid, and $\gamma_{\mathrm{fb}}$ is the interfacial tension between the film fluid and the bounding fluid.

Figure 2 shows a large drop of fluid on solid substrate in presence of bounding fluid and the interfacial tensions at the three phase contact line. A negative spreading coefficient $S$ implies partial wetting or dewetting, which results in destabilization of the thin film.

2.1.2. Evolution of Instability. The following long-wave equation describes the 2D dynamics and shape of a thin fluid film on a solid substrate (here, the gravity effects and evaporation/condensation are neglected):

$$
\begin{array}{r}
3 \eta \frac{\partial h(x, t)}{\partial t}+\frac{\partial}{\partial x}\left[\gamma_{\mathrm{fb}} h^{3}(x, t) \frac{\partial^{3} h(x, t)}{\partial x^{3}}\right] \\
-\frac{\partial}{\partial x}\left[h^{3}(x, t)\left(\frac{\partial^{2} \Delta G}{\partial h^{2}}\right) \frac{\partial h}{\partial x}\right]=0, \\
\frac{\partial H}{\partial T}+\nabla \cdot\left[H^{3} \nabla\left(\nabla^{2} H\right)\right]-\nabla \cdot\left[H^{3} \Phi \nabla H\right]=0, \\
\text { nondimensional equation. }
\end{array}
$$

Here, $h(x, t)$ is the local film thickness.

A linear solution of (4) can be written as follows

$$
h(x, t)=h_{0}(x, t)+\delta \operatorname{Exp}[q x] \operatorname{Exp}[R t]
$$

$$
\Delta G=S_{c}^{\mathrm{LW}} \frac{d_{0}^{2}}{h^{2}}+\left(S_{s}^{\mathrm{LW}}-S_{c}^{\mathrm{LW}}\right) \frac{d_{0}^{2}}{(h+\delta)^{2}}+S_{k}^{p} \operatorname{Exp}\left[\frac{\left(d_{0}-h\right)}{l_{0}}\right]
$$

A 3D plot of the solution is, as shown in Figure 3, computed in Mathematica Software. The diagram clearly indicates the emergence of spines at the early stage of spinodal dewetting where linear solution is taken.

Coordinate $x$ is parallel to the substrate surface, $t$ is time, $\eta$ is viscosity, $\gamma_{f}$ is the surface tension of the film, and $\Pi$ is the disjoining pressure of the film which is related to the total excess free energy $(\Delta G)$ of the film per unit area due to various types of intermolecular interactions

$$
\Pi=-\left(\frac{\partial \Delta G}{\partial h(x, t)}\right) \text {. }
$$

For thinner films intermolecular forces get more dominant. Their importance is manifested for example for van der Waals forces by the disjoining pressure which expresses the tendency of the film to adjust its thickness in order to minimize its free energy. On the other hand, the surface tension tries to keep the film flat. This is expressed by the Laplace pressure. In the case of a nonvolatile liquid, disjoining and Laplace pressure determines the shape of the film on a solid substrate

$$
\text { Laplace pressure } P_{L}=\gamma\left(\frac{d^{2} h(x, t)}{d x^{2}}\right),
$$


and for nonretarded van der Waals force the disjoining pressure is given by:

$$
\Pi=-\frac{A}{6 \pi h^{3}} .
$$

Here, $\gamma$ is the interfacial tension of film-bounding interface and $A$ is an effective Hamaker constant. The sign of $A$ tells us if molecules of the film are more attracted by like molecules or by the substrate. If $A$ is negative the film shows the tendency to get thinner. Equation (4) is the sum of the three forces per unit width of the film: the first term on the left hand side is the viscous force, the second term is the surface tension force due to the curvature at the free interface, and the last term is the sum of excess intermolecular forces. The viscous merely retards the growth of the instability, whereas the interfacial surface tension force has a stabilizing influence until the film ruptures. The surface tension wants to keep the film flat so that it can have minimum area and thus minimum excess surface free energy before the rupturing of the film. After the appearance of a hole, the surface tension assists in the growth of the hole

$$
-\frac{\partial^{2} \Delta G}{\partial h^{2}}=\frac{d \Pi}{d h}
$$

$d \Pi / d h>0$ can endanger the interfacial stability by spinodal decomposition manifested as the growth of thicker regions of the film at the expense of the thinner regions.

2.1.3. Excess Free Energy of the Film. The total excess free energy of the film is derived from the long-range apolar Lifshitz-van der Waals (LW) interaction and from the shorter-ranged polar (e.g., acid base) interactions which become significant for hydrogen bonding substances like aqueous systems.

The LW component of the excess interaction potential per unit volume, $\phi^{\mathrm{LW}}$ is made up of six different LW interactions as (1) interactions among the molecules of the film of thickness $h$, (2) interactions among the molecules of the film and the coating of thickness $\delta$, (3) interactions among the molecules of the film and the bulk substrate, (4) interactions among the molecules of the film and overlying bulk bounding fluid, (5) interactions among the molecules of the bounding medium and coating, (6) interactions among the molecules of the bounding medium and the substrate.

The total excess LW potential $\Phi^{\mathrm{LW}}=\partial \Delta G^{\mathrm{LW}} / \partial h$ is given by the following expression in the microscopic approach of London and Hamaker:

$$
\begin{aligned}
\Phi^{\mathrm{LW}}= & \frac{\left(A_{f f}+A_{c b}-A_{f b}-A_{c f}\right)}{6 \pi h^{3}} \\
& +\frac{\left(A_{c f}+A_{s b}-A_{c b}-A_{s f}\right)}{6 \pi(h+\delta)^{3}},
\end{aligned}
$$

where $A_{i j}$ refers to the Hamaker constant in Joules for Lifshitz-van der Waals interactions between molecules of type $i$ and $j$ and subscripts $f, c, s$, and $b$ denote the film, coating, substrate, and bounding fluid materials, respectively.
The excess potential vanishes for a thick film $(h \rightarrow \infty)$ and reduces to the well-known form $A_{e} / 6 \pi h^{3}$ in the limits of $\delta \rightarrow 0$ (uncoated substrate) and $\delta \rightarrow \infty$ (thick coating).

2.2. The Theory of Spinodal Decomposition. Spinodal decomposition is the process of unmixing, that is, phase separation, which takes place in thermodynamically unstable solid solution. Examples which have been experimentally studied are $\mathrm{Al}-\mathrm{Zn}, \mathrm{Al}-\mathrm{Fe}, \mathrm{Zn}-\mathrm{Cu}$, and so forth. Each of these systems consists of a single uniform phase at sufficiently high temperatures and decomposes into a two-phase system when quenched. First appears a fine uniform dispersed precipitate, which subsequently coarsens and develops into distinct regions of coexisting equilibrium phases.

Few essential characteristic features of the spinodal mechanism are as follows.

(1) It is an unstable mechanism in contrast to nucleation which occurs in the metastable region of the miscibility gap. Spinodal decomposition requires no activation energy.

(2) The order parameter describing a spinodal system obeys a local conservation law. Generally, the order parameter in the system is the local concentration of one of the components of the system. The decomposition is limited by diffusion. The late stage coarsening (often called Ostwall ripening) proceed increasingly slowly, because system requires diffusion of material across larger and larger distances. This process is in contrast with the rapid approach to completion, which occurs in magnetic and structural phase transformations. For going to the mathematical analysis of spinodal decomposition, two further assumptions are needed for the simplification of the problem.

(3) The spinodal decomposition is assumed to occur as an isothermal process. Each point in the system is assumed to be in strong contact with a heat reservoir. There is no constraint of local energy conservation.

(4) The spinodal process is assumed to occur coherently, which means that in a crystalline system, the two coexisting phases exist on the same lattice.

2.2.1. The Thermodynamic Theory of Spinodal Decomposition: The Cahn Hilliard Equation. The states of the system can be described by a single function $c(r)$, which can be taken as the average concentration around position $r$ of one of the components of a binary solution. For each $c(r)$, there exists a coarse-grained Helmholtz free energy $F\{c(r)\}$.

In principle, $F$ can be calculated from a microscopic model as follows:

$$
\text { A configurational sum yields } \sum_{c_{i}(r)} \operatorname{Exp}\left[-\frac{F\{c(r)\}}{k_{B} T}\right] .
$$

Here, $T$ is the temperature, $k_{B}$ is the Boltzmann's constant, and $E$ is the energy of a microscopically specified configuration of the system. The constrained sum is 
performed over all microscopic configurations such that the average concentration in a course graining volume at position $r$ is $c(r)$. The specified form of $F$ which was chosen by Cahn and Hilliard is the familiar Gingburg-Landau functional

$$
F\{c\}=\int\left[\frac{1}{2} K(\nabla c(r))^{2}+f\{c(r)\}\right] d r .
$$

The functional $f\{c(r)\}$ is the free energy density for a uniform system and is assumed to exhibit two minima as shown in Figure 4.

$\delta F / \delta C(r)$ is the chemical potential gradient at the point $r$. The quantity $\delta F / \delta c$ is a thermodynamic force which drives an interdiffusion flux $\nabla J$. Let $M$ denote the linear response coefficient.

\subsubsection{Fourier Modes of Evolution of Instabilities}

$$
j(r)=-M \nabla \frac{\delta F}{\delta c(r)}=-M \nabla\left[K \nabla^{2} c+\frac{\partial f}{\partial c}\right] .
$$

Then, the generalized diffusion equation obtained from the continuity equation,

$$
\frac{\partial c(r)}{\partial t}=-\nabla \cdot j=\nabla \cdot M \nabla\left[K \nabla^{2} c+\frac{\partial f}{\partial c}\right]
$$

The above equation is known as the Cahn-Hilliard equation. A linearized solution of the above equation can be written as

$$
c(r, t)=c_{0}+u(r, t) .
$$

Now

$$
\frac{\partial u}{\partial t}=M \nabla^{2}\left[K \nabla^{2}+\frac{\partial^{2}}{\partial c^{2}}\right] u
$$

If $c_{0}$ is chosen to be equal to one of the equilibrium concentrations, say $C_{\alpha}$

$$
\frac{\partial^{2} f}{\partial c^{2}}=\frac{K}{\xi_{\alpha}^{2}}
$$

where $\xi$ is the correlation length in phase $\alpha$. Spatial variations of $u$ can be considered very slowly varying on the microscopic scale. Then,

$$
\frac{\partial u}{\partial t}=D_{\alpha} \nabla^{2} u, \quad D_{\alpha}=M \frac{\partial^{2} f}{\partial c_{\alpha}^{2}} .
$$

Let

$$
\begin{gathered}
u(r, t)=\operatorname{Exp}[i(K \cdot r-\omega t)] \\
\omega(k)=M k^{2}\left[K k^{2}+\frac{\partial^{2} f}{\partial c^{2}}\right]=M K k^{2}\left(k^{2}-k_{c}^{2}\right) .
\end{gathered}
$$

Here,

$$
k_{c}^{2}=\frac{1}{K}\left[\frac{\partial^{2} f}{\partial c^{2}}\right]_{c_{0}} .
$$

Composition fluctuations in the region $0<K<K_{c}$ are unstable and grow exponentially with time

$$
K=K_{P}=\frac{K_{c}}{\sqrt{2}},
$$

is the fastest growing mode which Cahn has identified as characterizing the initial spinodal instability determining the scale of emerging precipitates.

2.2.3. Stability of Stationary Configuration. Let $C_{s}(r)$ denote a general stationary solution. Then, the chemical potential should be constant over the region,

$$
\frac{\delta F}{\delta c(r)}=-K \nabla^{2} c_{s}+\frac{\partial f}{\partial c_{s}}=\mu=\text { constant. }
$$

For one-dimensional variations of $C_{s}$ in $+X$ direction,

$$
K \frac{d^{2} c_{s}}{d x^{2}}=-\frac{d}{d c_{s}}\left[-f\left(c_{s}\right)+\mu c_{s}\right] .
$$

The above equation is analogous to the motion of a particle mass of $K$ with displacement $C_{s}$ moving as a function of time $x$ in the potential $\left\{-f(c)+\mu c_{s}\right\}$. As long as this potential has a local minimum, there will be bounded solutions $C_{s}(x)$ with nontrivial $x$ dependence. These solutions will be periodic in $x$. The most important solutions are those in which the particle spends most of the time sitting near the peaks of potential (the minimum ( $f-\mu c_{\alpha}$ and $f-\mu c_{\beta}$ ) but periodically makes a transition from one peak to the other, thus describing a coarse-grained phase separated configuration.

2.3. Sinusoidal Composition Modulations (Fourier Modes) Leading to Phase Separation. John W. Cahn has predicted the structures in phase separation from a single-phase fluid by spinodal mechanism which can be described in terms of a superposition of sinusoidal composition modulations of fixed wave lengths, but random in amplitude, orientation, and phase.

Let us consider an inhomogeneous solution whose composition everywhere differs only slightly from the average composition. Its free energy is given by

$$
F=\int\left[f(c)+K(\nabla c)^{2}\right] d v
$$

Here, $f(c)$ is the free energy density of homogeneous material of composition $c . \nabla c$ is the additional free energy density if there is concentration gradient in composition. The gradient of the compositions are taken to be small. $f(c)$ can be expanded about the average composition $C_{0}$

$$
f(c)=f\left(c_{0}\right)+\left(c-c_{0}\right)\left(\frac{\partial f}{\partial c}\right)+\frac{1}{2}\left(c-c_{0}\right)^{2}\left(\frac{\partial^{2} f}{\partial c^{2}}\right)+\cdots
$$

(H. order term). 


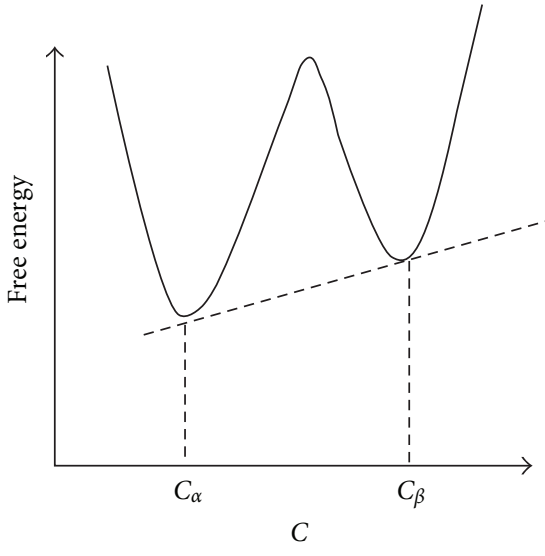

(a)

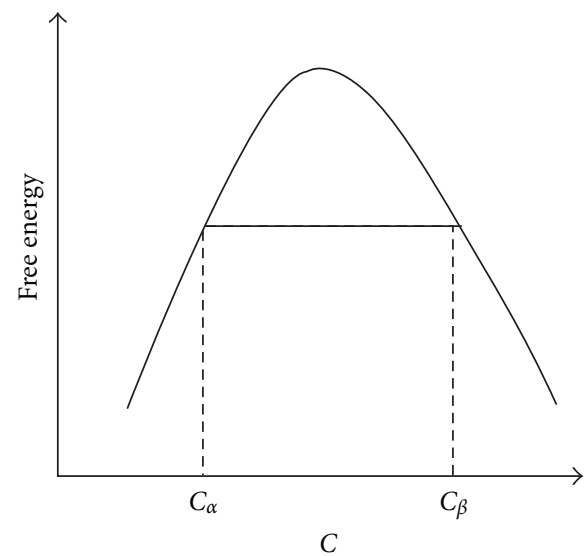

(b)

FIGURE 4: Free energy diagram of a binary mixture (A-B-alloy).

From mass conservation, one can write

$$
\int\left(c-c_{0}\right) d v=0
$$

We can express the difference between the free energy of initially homogeneous solution and the inhomogeneous solution as

$$
\Delta f=\int\left[\frac{1}{2}\left(\frac{\partial^{2} f}{\partial c^{2}}\right)\left(c-c_{0}\right)^{2}+K(\nabla c)^{2}\right] d v .
$$

It is clear from the (29) that the solution is stable to all infinitesimal fluctuation if $\partial^{2} f / \partial C^{2}>0$, whereas if $\partial^{2} f / \partial C^{2}$ is negative, the solution is unstable to such a fluctuation for which the first term dominates. This condition can be always fulfilled if the spatial variation in the density of the composition is very slow.

The composition can be considered of several Fourier components. $\Delta F$ is the sum of contributions from each Fourier components separately.

Let us consider one such component

$$
c=A \cos \beta x .
$$

The contribution of this to the change in the free energy $\Delta F$ is the quantity

$$
\begin{gathered}
\frac{1}{4} V A^{2}\left[\left(\frac{\partial^{2} f}{\partial c^{2}}\right)+2 K \beta^{2}\right], \\
\int \cos ^{2} \beta x d x=\frac{1}{2} .
\end{gathered}
$$

Thus, the Fourier components of sufficiently large wave length, that is, sufficiently small wave number $\beta$, result in decreasing free energy. If the solution is in the unstable region; the maximum wave number $\beta_{c}$ is given by

$$
\beta_{c}=\left(-\frac{\partial^{2} f / \partial c^{2}}{2 k}\right)^{1 / 2}
$$

The kinetics of the initial stages of phase separation is obtained by simply solving the diffusion equation

$$
J_{\beta}=-J_{\alpha}=M \nabla\left(\mu_{\alpha}-\mu_{\beta}\right) .
$$

Here, $J$ is the current density in diffusion under influence of effective field $E$ at some instant, $c$ is the average concentration at the locality, and $M$ is defined as the mobility

$$
J=M \nabla\left(\mu_{\alpha}-\mu_{\beta}\right) \text {. }
$$

$M$ must be positive for diffusion to take place, which results spontaneously from the chemical potential gradient in order to minimize the free energy

$$
\begin{aligned}
\left(\mu_{\alpha}-\mu_{\beta}\right) & =\left(\frac{\delta F}{\delta c_{\alpha}}\right) \\
& =\left(\frac{\partial f}{\partial c_{\alpha}}\right)-2 K \nabla^{2} c_{\alpha}+\text { higher order terms. }
\end{aligned}
$$

Substitution gives

$$
\frac{\partial c}{\partial t}=M\left(\frac{\partial^{2} f}{\partial c^{2}}\right) \nabla^{2} c-2 M K \nabla^{4} c .
$$

The solution to (37) can be written as

$$
c-c_{0}=\operatorname{Exp}[R(\beta) t] \cos (\beta \cdot r) .
$$

Here, $R(\beta)$ is given by

$$
R(\beta)=-M\left(\frac{\partial^{2} f}{\partial c^{2}}\right) \beta^{2}-2 M K \beta^{4} .
$$

$R(\beta)$ is negative for all $\beta$ in the metastable region and positive for $\beta<\beta_{C}$ in the unstable region. Because the equation is homogenous and linear in $c$, all sums of solutions are also possible solutions

$$
\begin{aligned}
c-c_{0}= & \sum_{\operatorname{all} \beta} \operatorname{Exp}[R(\beta) t] \\
& \times[A(\beta) \cdot \cos (\beta \cdot r)+B(\beta) \cdot \sin (\beta \cdot r)] .
\end{aligned}
$$




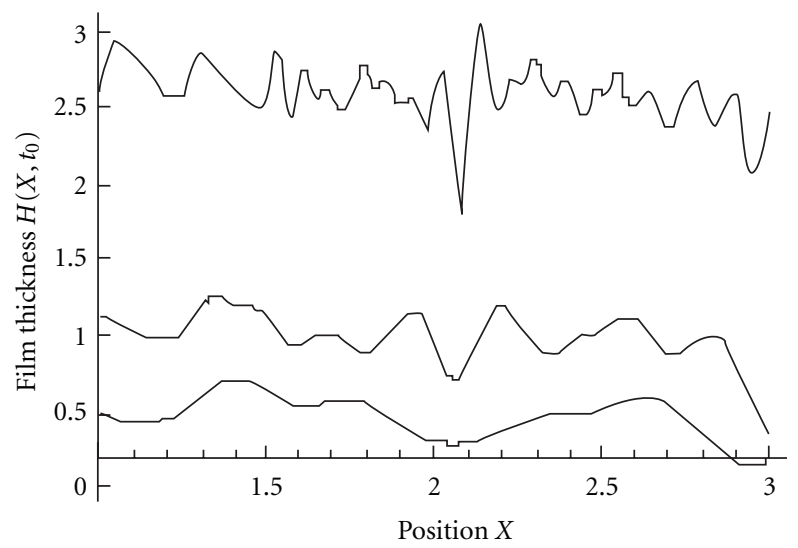

FIgure 5: The figure shows amplitude modulations in spinodal decomposition perturbations with higher harmonics show typical variation in it.

The amplification factor $R(\beta)$ has a sharp maximum at

$$
\beta_{m}=\frac{\beta_{c}}{\sqrt{2}}=\frac{1}{2}\left[-\left(\frac{\partial^{2} f}{\partial c^{2}}\right) \frac{1}{K}\right]^{1 / 2} .
$$

All other Fourier components other than $R\left(\beta_{m}\right)$ can be neglected in the exponent. Thus,

$$
c-c_{0} \cong \operatorname{Exp}\left[R_{m}\right] \sum_{\beta_{m}} \beta_{m}[A(\beta) \cdot \cos (\beta \cdot r)+B(\beta) \cdot \sin (\beta \cdot r)] .
$$

At some time after phase separation, composition in the solution becomes a superposition of sinusoidal waves of fixed wavelength but random in orientation, phase, and amplitude. The initial stages of phase separation are symmetric about $c_{0}$. As the phase separation continues, higher-order terms in (29) will bring in harmonics which will distort the composition as a consequence. The resultant structure will become a mixture of two homogeneous phases.

In this discussion of theory, the contribution of surface free energy is implicit. This assumes that the geometrical arrangement of the composition modulation does not change with time during the early stage process. Only the amplitudes of the modulations change Figure 5.

\section{Methodology and Computational Procedure}

3.1. Surface Directed Spinodal Decomposition: Model and Simulation Geometry. Computer simulation is done with two films A and B, respectively, composed of spin +1 and spin -1 . The majority component spin down $(-1)$ and minority component spin up $(+1)$ were initially placed randomly on the different lattice points. The two substrates exhibits strong repulsive interaction with type A component $($ spin +1$)$ and strong preferential attraction for type B film (spin -1$)$ as shown in Figure 6.

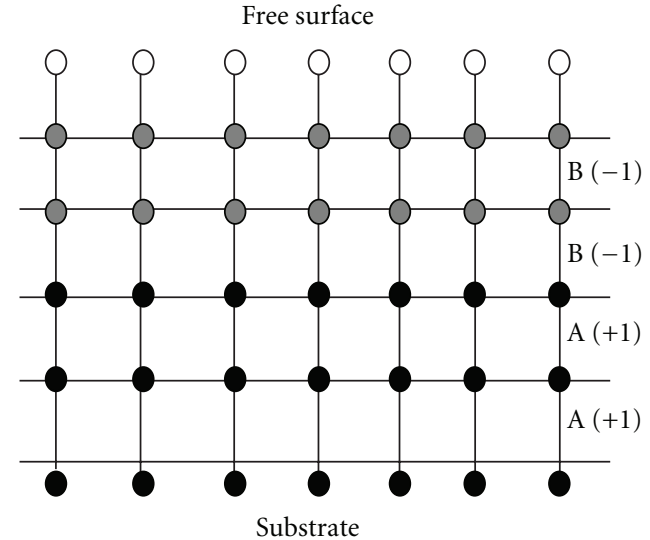

FIGURE 6: Shows the initial configuration of type A $($ spin +1$)$ and type $B($ spin -1$)$ with respect to the substrate. In this paper, we have started with a random distribution of A-B in 30:70 ratio confined between two walls with strong preferential attraction to one the component B in our case and repulsive to another component in minority in this case A. Peroidic boundary conditions are applied in $X$ direction.

The energy of the system in the configuration $\left\{S_{i}\right\}$ is defined as

$$
E\left\{S_{i}\right\}=-\sum_{\substack{\langle i j\rangle \\ i \neq j}} \varepsilon_{i j} S_{i} S_{j},
$$

where $\varepsilon_{i j}$ is negative for antiferromagnetic interactions and $S_{i}$ and $S_{j}$ represent the spin states.

Thus, the partition function for the system can be written as

$$
Q(H, T)=\sum_{\left\{S_{i}= \pm 1\right\}} e^{-\beta E\left\{S_{i}\right\}},
$$

where $\beta=1 / k_{\beta} T$.

The probability $P\left(X_{\lambda}\right)=P(X, t)$ has been considered with a characteristic length scale of $\lambda$ which represents the probability of the configuration $X$ at time $t$ in the Monte Carlo process. The process satisfies the Markovian master equation as follows [37]:

$$
\begin{aligned}
\frac{d P(X, t)}{d t}= & -\sum_{X} W\left(X \longrightarrow X^{\prime}\right) P(X, t) \\
& +\sum_{X^{\prime}} W\left(X^{\prime} \longrightarrow X\right) P\left(X^{\prime}, t\right) .
\end{aligned}
$$

In (45), the first term on the right hand side represents all processes where the system moves away from the considered state $X$, and hence its probability decreases. The second term contains all reverse processes, and it leads to an increase of the probability of finding state $X$.

The detail balance condition always ensures that the sum of the above two terms on right hand side of (45) cancels each other so that equilibrium is achieved for which

$$
P(X, t)=P_{\mathrm{eq}}(X) \Longrightarrow \frac{d P(X, t)}{d t}=0 .
$$


The probability of getting $X_{i+1}$ from $X_{i}$ is defined as the transition probability $W\left(X_{i+1} / X_{i}\right)$ and is chosen such that the ultimate distribution of states $X_{0}, X_{1}, \ldots, X_{n}$ is a Boltzmann distribution and is given by $P\left(X \rightarrow X^{\prime}\right)$ as follows:

$$
P\left(X \rightarrow X^{\prime}\right) \propto e^{-\left[\Delta E / k_{B} T\right]} .
$$

$P\left(X \rightarrow X^{\prime}\right)$ is called the metropolis function. Here $X$ and $X^{\prime}$, respectively, correspond to $X_{i}$ and $X_{i+1}$. In the present case, the change in energy $\Delta E=E\left(X_{i+1}\right)-E\left(X_{i}\right)$ is negative, hence $W\left(X_{i+1} / X_{i}\right)=1$. To avoid confinement at a local minimum, moves with low probabilities have also been accepted occasionally which raises the energy

$$
W\left(\frac{X_{i+1}}{X_{i}}\right)=\min \left[1, \exp \left(-\frac{\Delta E}{k_{B} T}\right)\right] .
$$

Glauber Kinetics function $\left[1 / 2\left(1-\tanh \left(\Delta E / 2 k_{B} T\right)\right]\right.$ has been chosen to calculate transition probability for achieving detail balance condition. Semi-infinite periodic boundary condition has been employed for the model system, because it is not plausible to work with infinitely large system to get correct picture of the time evolution. Rather, the boundary condition applied makes it possible to achieve the thermodynamic limit $N \rightarrow \infty$ [38]. Adoption of semi-infinite periodic boundary condition for $X$ direction of lattice means

$$
S(N+1, j)=S(1, j), \quad S(0, j)=S(N, j) .
$$

Random numbers have been generated using standard ran2 (idum) function program. Kawasaki spin exchange procedure is adopted in which each spin of the lattice has been chosen one by one in a regular manner and one of its 4 nearest neighbors has been taken corresponding to a random number. The free energy has been calculated by exchanging the two spins. If $P(x) \geq$ rand $(i)$, the exchange of the spins or the move has been accepted where rand $(i)$ is the corresponding random number generated in the process; otherwise, the spins are restored to their original positions. The equality does not allow the system to be trapped in any local minimum of free energy. All the computations have been carried out on a PC with Pentium Core Duo processor.

3.2. Spinodal Dewetting of Polymer Film: Model and Simulation Geometry. The polymer chains are described by $N$ effective monomers ( $N=24$ here), and beads are connected by springs representing "effective bonds" using the FENE (finitely extensible nonlinear elastic) potential for bond length $l$ as follows:

$$
U_{\mathrm{FENE}}^{l}=\frac{1}{2} k\left(l_{\max }-l_{0}\right)^{2} \ln \left[1-\frac{\left(l-l_{0}\right)^{2}}{\left(l_{\max }-l_{0}\right)^{2}}\right] .
$$

This potential has its minimum $U_{\mathrm{FENE}}^{l_{0}}=0$ for a bond length $l_{0}$. The potential is harmonic in the vicinity of $l_{0}$ and has the minimum value $U_{\mathrm{FENE}}^{l_{0}} \approx(1 / 2) K\left(l-l_{0}\right)^{2}$, where $k$ is the spring constant. The potential diverges logarithmically both for $l \rightarrow l_{\max }$ and $l \rightarrow l_{\min }=2 l_{0}-l_{\max }$. Thus, the length of the effective bond is restricted to the range $l_{\min }<l<l_{\max }$. Unit of length is defined such that $l_{\max }=1.2$, and other parameters are

$$
\begin{gathered}
l_{\min }=0.2, \quad l_{0}=0.7, \\
K=200.0 \quad \text { at } k_{B} T=1.8 .
\end{gathered}
$$

Here, the coarse-grained chains are treated as being fully flexible, and thus any conformational potential for bond angle or torsion of bonds are not included. Such a choice is reasonable here, because groups of $\mathrm{n}$ successive chemical bonds along the backbone of the chain are combined into one effective bond such that the length of effective bond is of the order of the persistence length (typically here, $n \approx$ 15-18) [39]. For these effective bonds the bond angle and torsion potentials that act on the level of chemical bonds are ignored. To compute nonbonded interaction between effective monomers, both on the same chain and on different chains, a Morse potential of strength $\varepsilon_{M}$ has been used given as follows:

$$
U_{M}^{(r)}=\varepsilon_{M} \operatorname{Exp}\left[-\frac{2}{\xi}\left(r-r_{\text {min }}\right)\right]-2 \varepsilon_{M} \operatorname{Exp}\left[-\frac{1}{\xi}\left(r-r_{\min }\right)\right] .
$$

At the $r_{\min }=0.5$, this potential has its minimum depth as $\varepsilon_{M}$. Clearly, the preferred distance between nonbonded ones is not the same as the preferred distance between neighboring bonded ones as $l_{0}$. Thus, a tendency to crystallization in dense configurations at low temperatures is avoided. The parameter $\alpha$ describes the inverse range of this potential. $\alpha=$ $1 / \xi$ is chosen to be large $(\alpha=9.0)$. This consequently makes the potential extremely short ranged. $U_{M}(r \rightarrow 1.0) \approx 0$ choosing $\varepsilon_{M}=1.0$

24 beads have been taken in each polymer chain along $X$ direction. 18 such chains have been placed in $Y$ direction and 6 chains one above other in $Z$ direction. As a simulation geometry, a $L_{X} L_{Y} L_{Z}$ size box with $L_{X}=78 \sigma, L_{Y}=58.5 \sigma$, and $L_{Z}=26 \sigma$ with $\sigma=0.2 A^{0}$ is chosen with two impenetrable walls at $z=0$ and $z=D$. In $\mathrm{X}$ and $Y$ direction, periodic boundary conditions are applied.

At the lower wall $(Z=0)$ an adsorption potential acts as follows:

For

$$
\begin{gathered}
z<\delta=\frac{1}{8.0}, \\
U_{\text {ad }}(z)=-\frac{1}{z^{3}} .
\end{gathered}
$$

For

$$
\begin{gathered}
z \geq \delta, \\
U_{\mathrm{ad}}(z)=0 .
\end{gathered}
$$

At $z=D, U=\infty, D$ the distance between two walls has been taken as $26 \sigma \AA$, where $\sigma=0.2$.

3.2.1. Adsorption Potential: Surface-Surface Interaction. The interaction energy of two surfaces at a distance $r$ apart is 
calculated here. For two infinite surfaces, the energy will be infinity, hence the energy per unit area is considered. The potential can be calculated by considering a thin sheet of molecules of unit area and thickness $d z$ at a distance $z$ away from an extended surface of large area.

The energy of interaction of this sheet with the surface can be given by (55) [40]

$$
u(r)=\frac{2 \pi C \rho(\rho d z)}{(n-2)(n-3) z^{n-3}} .
$$

Thus, for two surface, we have

$$
W(r)_{\text {surf-surf }}=\frac{2 \pi C \rho^{2}}{(n-2)(n-3)(n-4) r^{n-4}} .
$$

The microscopic van der Waal's energy between two molecules arising from the induced dipole-dipole interaction varies as $r^{-6}$ in the static approximation, $r$ being the separation of molecules. For larger distance $(>100 \mathrm{~nm})$, retardation effects have to be considered, and the interaction falls as $r^{-7} \cdot r^{-7}$ is the case applied while considering the adsorption of the polymer film on solid substrate, where substrate's thickness is effectively $>100 \mathrm{~nm}$. Then, one can write the adsorption potential

$$
U_{\text {ads }} \frac{\alpha 1}{z^{3}}
$$

Thus, here microscopic structure/atomic structure of the surface are zoomed out. Dynamics is associated to the model by choosing a single monomer by walking self avoid randomly. The linear chained polymer film is sandwiched between two walls, but lower wall has weak attractive force fields to the monomers. Self-avoid random walk has been performed keeping in view the fact that when temperature of the polymer is raised, chain segments within the long chain molecule are first mobilized before the whole molecule starts to move. In some parts within the molecule, there is a considerable localized motion in comparison to other parts of the same molecule. Some segments may have certain freedom of movement, whereas others do not have [41]. The attempted Monte Carlo update consists in a monomer displacement where the new position $r^{\prime}=r \pm \Delta r$ with $\Delta r=(\Delta x, \Delta y, \Delta z)$ being chosen randomly and uniformly from the intervals $-0.30 \leq \Delta x, \Delta y, \Delta z \leq 0.30$. The potentials described above ensure that no intersection of bonds can occur. The above displacement ensures a reasonably high acceptance rate of the move. The kinetics adopted in the simulation is the Glauber kinetics which better represents the slower processes. The Glauber kinetic function $0.5 *(1.0-$ $\tanh (\Delta E / 2.0 * k * T))$ [37] is always normalized between $0-1$, and thus it is easy to compare it with the stochastic probabilities between $0-1$. When any monomer is reached during the self avoiding random walk, that bead is displaced and is given new coordinates as $r^{\prime}=r \pm \Delta r$ as per a random number rand $(i)$ generated between 0.0 and 1.0. The change in free energy is evaluated and if the Glauber function is found to be $\geq$ rand $(i)$, the move is accepted otherwise that bead is moved to its original position.

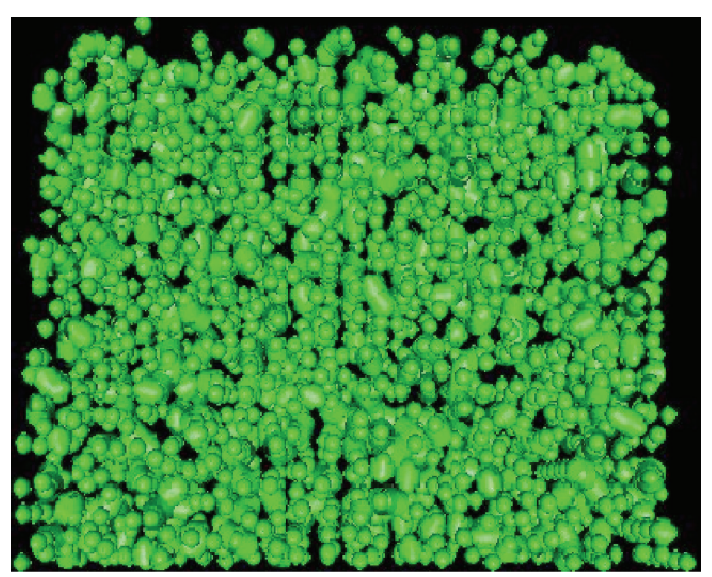

FIGURE 7: Shows micrograph after 2000 Monte Carlo cycles (3D NVT-MC simulation of linear polymer). Circular holes are created in the polymer film caused by rupturing mechanism (i.e., spinodal dewetting). The polymer film is sandwitched between two hard walls. $k_{B} T=1.8$ and sping constant of the film are taken as $K=200$. Metropolis function has been used by performing a complete self avoiding random walk on the beads of the chains. FENE potential has been used for intrachain beads and Morse potentail for interaction between inter- and intrachain beads. The lower substrate has weak attractive potential to the beads. Peroidic boundary conditions are applied in $X$ direction. Program has been developed by the author in $\mathrm{f} 77$.

A photomicrograph of early stage dewetted pattern is as shown in Figure 7 as obtained by the author by a 3D NVTMC simulation for a thin polymer film confined between two parallel hard walls. The substrate has an weak attractive interaction with the monomers. The upper hard wall is kept two layers above the initial configuration of the thin polymer film. Polymer beads have FENE potential with each other on the same chain and Morse potential with inter- and intra-chain beads. A self-avoiding random walk is performed during the simulation, and periodic boundary conditions are applied in $X$ and $Y$ directions. The number of holes created in film increases with run time. The average bond length increases rapidly for first few cycles when swelling and melting of the film is dominant. Later, the average bond length increases gradually following linear growth in early stages of the simulation. Confinement in $Z$ direction gives rise to dominant emergence of spines in $X-Y$ plane which can be accounted as hard repulsions at boundaries can be propagated in $X-Y$ plane because of the confinement in $Z$ direction. Average bond length has been observed to increase rapidly for first few cycles during swelling and melting of polymers and then gradually increases linearly $[19,20]$ as a consequence of the interplay of the forces between surface and molecular interactions of the beads of the polymer films. The number of circular holes created in the process increases with the increase in run time which we can believe to be a consequence of emergence of spines as no external heterogeneous influences can be present. Figure 8 shows a micrograph of surface-directed phase separation with one phase (minority). A spinup $(+1)$ is having repulsive 
interaction with the substrates. The 2D MC simulation has been performed using semi-infinite boundary conditions, Metropolis algorithm and Kawasaki spin exchange model with conserved spins on a lattice of $200 \times 100$ with $70: 30$ concentrations of spindown and spinup at $T=0.70[2$, $3]$. Both the substrate and the upper surface have strong preferential attraction to one of the component spin down $(-1)$ in this paper and strong repulsive force to the other component in minority here in this case spin up $(+1)$. The micrograph is isotropic and shows a clear signature of rich regions near walls and segregation of domains in preferably normal direction to the two surfaces as can be observed in Figures 8 and 9. Any isotropic or correlated or both together can lead to a direct deviation from Poisson distribution. Spinodal rupturing and creation of holes have been experimentally shown to fit for Poisson distribution but those are the cases not for such enforced or confined system which could enhance lateral modes of relaxation. Though the two processes as spinodal dewetting of thin films and surface-directed phase separation are phenomena of different temperature regimes, but the phenomenology of the evolution of the instabilities is common causing rupturing in the films. Using Binder and Sadiq inverse interfacial energy formula, two different regimes for growth of domain are observed $[2,3]$. In early stages when nonlinear and higher-order terms are not dominant, it would be safe to make qualitative comparison of the micrographs based on analytical expressions which indeed allows one for the same as can be learnt from the qualitative mathematical formulation of the spinodal theory applicable to both processes given in this paper.

\section{Conclusion and Remarks}

It can be observed that spinodal dewetting of thin films and surface-directed phase separation show significant similarities in their evolution justifying their common spinodal rupturing mechanism. This paper work indicates for the lateral and global nature of correlations during evolution under confined geometries which can be learnt from the result given in Figure 9. The confinement and surface fields can be expected to enhance the particle-particle or spinspin spatial correlations [42]. We observe correlated and anticorrelated behavior of the two components which have some oscillating character in the evolution of the system which can be accounted to the driving force fields of the two substrates and interplay with the locally dominant phase separation force field as per equation (25). There are several phenomena in physics, which are well explained by spring models for solids. The van der Waals forces in solids may be considered to be more significant. Relatively more complex nanostructures involving combination of holes, islands, and rims are witnessed in the instability of thin liquid films in 3D. Researchers doing simulations in this area have reported that dewetting is a very sensitive phenomenon (i.e., sensitive to the forces at work) as the results of the simulations with single and double precision are quite different in nature [36]. More kinetic studies of thin film breakup are still required to fully establish the spinodal dewetting. We need a detailed

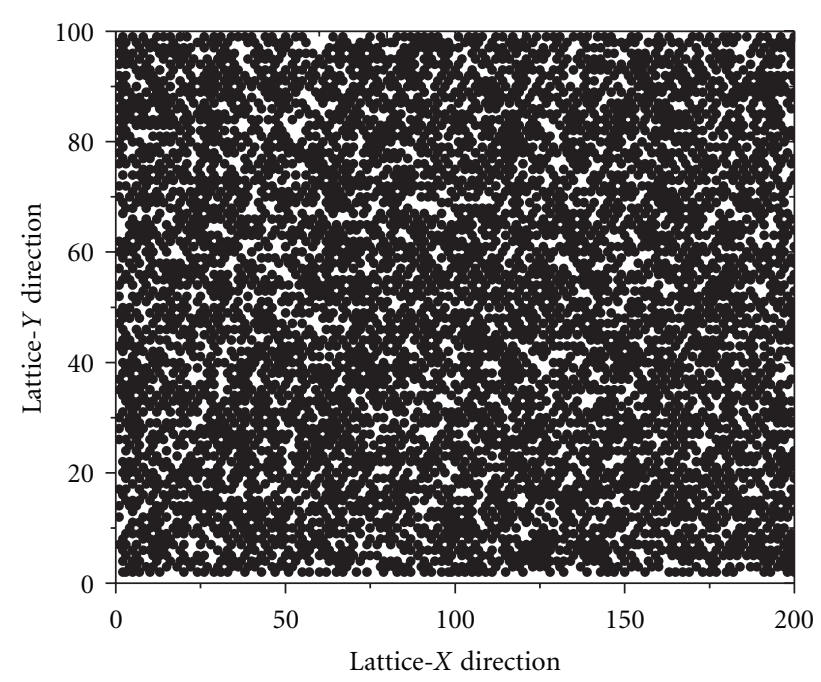

Figure 8: The figure shows micrograph of minority component after 30,000 Monte Carlo cycles (NVT-2D simulation). The Metropolis algorithm has been used in a self developed program with lattice size as $200 \times 100$ with $70 \%: 30 \%$ compositions of the preferred and unpreferred components. The majority component has strong affinity to the two confining substrates and the minority experiences strong repulsive force field of the two substrates. Periodic boundary condition is applied in $X$ direction. Program has been developed by the author in $\mathrm{f} 77$.

mechanism for explaining regular patterns originated from initially randomly distributed holes, says G. Reiter. A rigorous mathematical theory for dewetting is still awaited. Spinodal dewetting of thin polymer films are observed above their glass-transition temperature, which is quite high even in comparison to room temperature. The dipole-dipole (directional) interactions are linearly polarized so that it restricts the system to develop a perfect global minimization of free energy or say the system can not behave as a super molecule. The evolution of patterns can be a consequence of complex behavior of superposed/overlapped and nonoverlapped spinodal states [27], of course not resolved in the present existing literature of spinodal dewetting problems to the best knowledge of the author. The spinodal dewetting far below zero degree Celsius has not been observed in the case of inert gases films as no possible applications are known but that could be of interest for understanding the fundamentals of the interactions at molecular scale. van der Waal's forces between atoms without permanent dipoles may be described as resulting from the interactions of multipoles associated quantum transitions of the atoms. When the atoms are far apart from each other, only the dipole-dipole interactions are appreciable, but for the distances of the order of the radius (from kinetic theory), higher poles cannot be neglected, and their interactions should be taken into account. Henry Margeau examined and evaluated three terms of the series of interactions as (1) dipole-dipole interaction, (2) dipolequadrupole interaction, and (3) quadrupole-quadrupole interaction in his paper [43]. Two types of interactions are taken into account for nonpolar, unexcited atoms: repulsive 


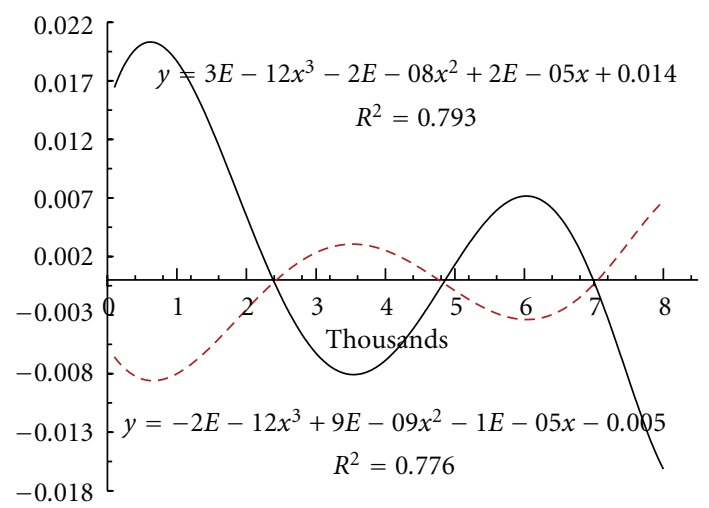

(a)

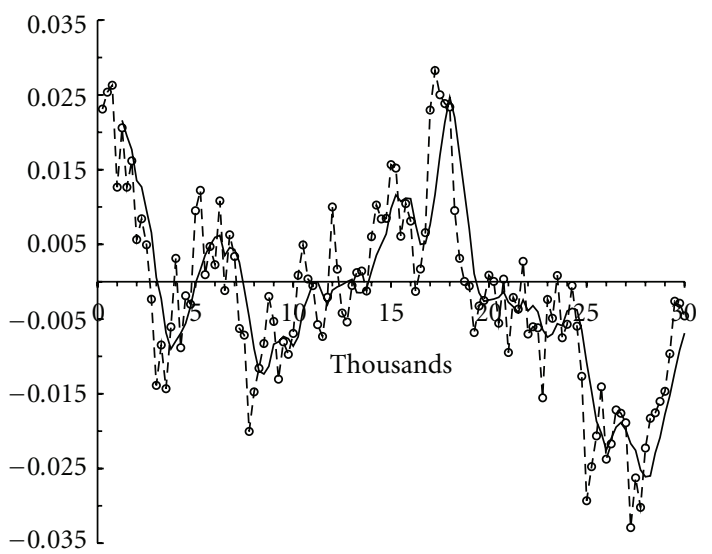

(c)

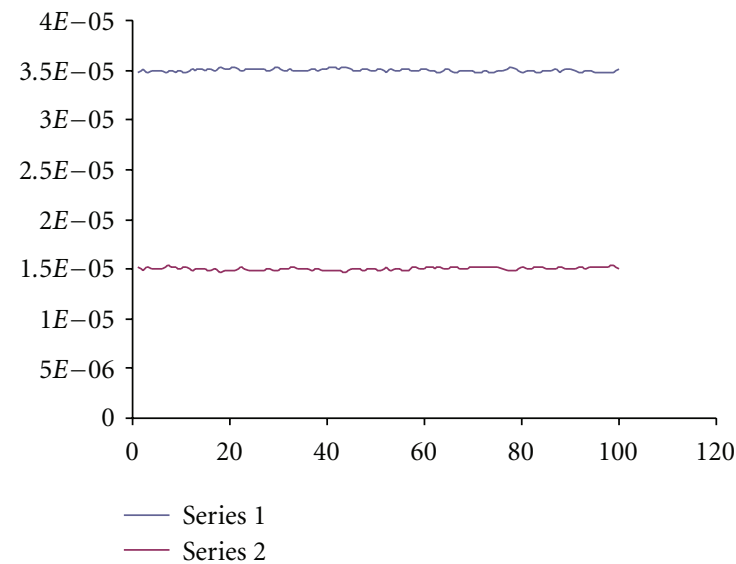

(e)

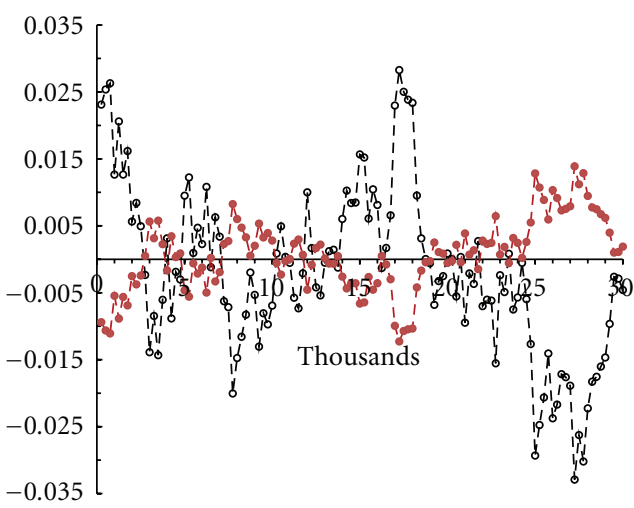

$-\bullet-$ Series 1

- - Series 2

(b)

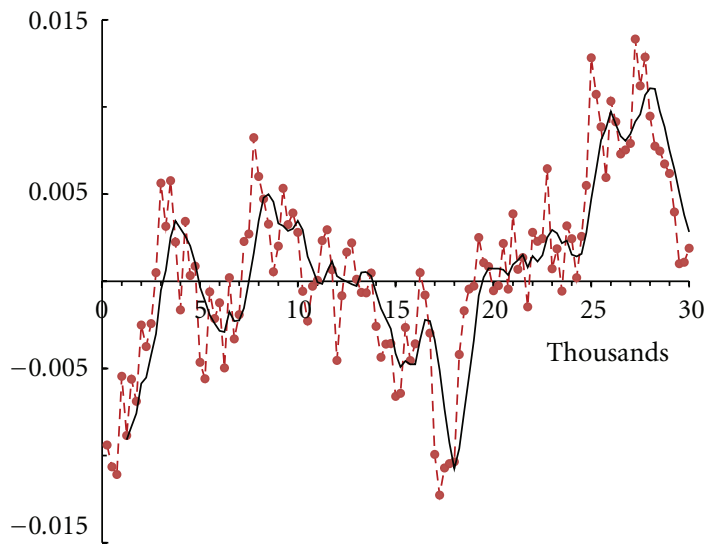

(d)

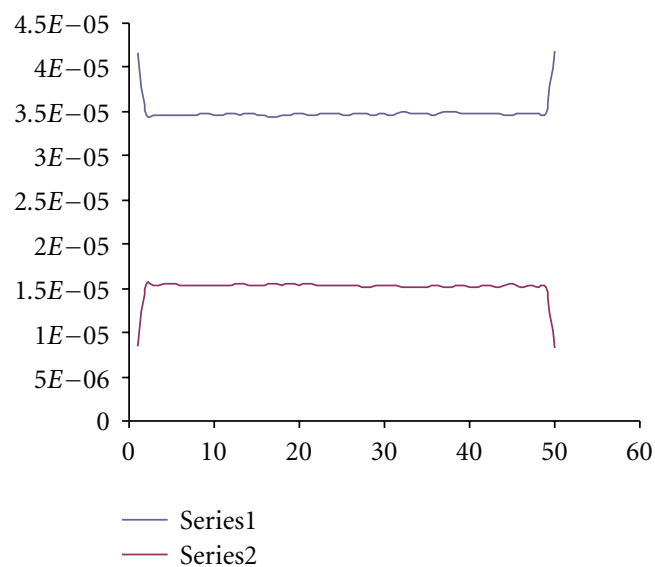

(f)

FIGURE 9: It shows (a) spatial correlation fits well to higher order polynomial specially in early phases of evolution; spatial correlation versus MC cycles (b) spatial correlation versus MC cycles, the open dots represent the preferred component B (i.e., majority component) and solid ones the other one (i.e., minority component-A) (c) spatial correlation of B (average fitting) versus MC cycles (d) spatial correlation of A (average fitting) versus $\mathrm{MC}$ cycles (e) average number density of A and B-profile parallel to the substrates and (f) average number density of A and B-profile perpendicular to the substrates. All averages are done after every 250 cycles (except (a) after $100 \mathrm{MC}$ cycles). 


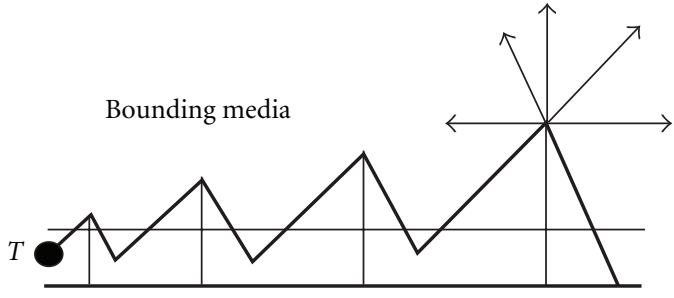

FIGURE 10: The figure shows a sketch of asymmetric interface formation on a weakly wettable surface or rod. A net potential gradient can provide a driving force. Such microstructures or nanostructures can also lead to the self diffusion of biological molecules, liquid crystals, and so forth in various ambient conditions.

forces due to electrons and attractive polarization forces. The repulsive forces fall of sharply near the gas kinetic boundary of atom, while the attractive polarization forces have a much larger range and predominate in the region, where the wave function of the atom is small. To calculate both types of forces by means of a coherent process of approximations is a difficult task in general and has been worked out only for hydrogen.

In his paper, Margenau showed that while higher order pole interactions may be neglected, the interactions between dipoles and quadrupoles furnishes an appreciable contribution to the van der Waal's forces. The present work on spinodal dewetting of existing thin film systems does not include these discussions in some detail, which in fact can provide new routes of free energy minimization and may also lead to new kind of structure formations if the parameters are properly tuned in. In fact, it is well known that breathing effect through nucleus have been observed in spin relaxation processes in case of dielectric phenomena, but the effect of dipole-quadrupole has been not yet discussed or found any attention in scientific realm when describing similar processes of relaxation as spinodal dewetting of thin nanofilms which takes place in a time window of few nanoseconds in case of metal films and of several hours in case of polymer films and any colossal discussion or theory is not available.

For making a polymeric nano/micromotor, its one end would be required to be attached with a bead (Figure 10), which can be thermally more susceptible to the radiations in comparison to the other segments of the macromolecule. An induced dipole at $\mathbf{T}$ would induce dipole next to it, and thus induced dipole-induce dipole interaction can prevail via Van der Waals force and would amplify it if that minimizes the energy in the bounding media. An asymmetric geometry of spines when attached to a rod would provide a net interfacial tension in the bounding media and would drive it. It has been found that similar asymmetric potential gradient can drive nanomotors. Liquid crystals do have strained structures. Such type of driving mechanism has not been done and implemented yet. The experimental tools are still far from reality by at large intrigue into problems discussed as earlier at very small scale ranging from mesoscale to nanoscale, and one may require new modifications [44] in experiments to independently investigate and match with the theoretical predictions using computational methods within the regression of the existing theories and based on the proven foundations of physics.

\section{References}

[1] S. Herminghaus, K. Jacobs, K. Mecke et al., "Spinodal dewetting in liquid crystal and liquid metal films," Science, vol. 282, no. 5390, pp. 916-919, 1998.

[2] S. P. Singh, K. Singh, and M. Roychoudhury, "Monte Carlo simulation for diffusion limited surface directed phase separation," Proceedings of National Academy of Sciences A, vol. 78, pp. 79-83, 2008.

[3] S. P. Singh, "A review of molecular simulation: future scope and limitations ref-583," in Proceedings of the International Conference on Nanomaterials and Nanotechnology, Erode Tamilnadu, India, December 2010.

[4] A. Sharma and R. Khanna, "Pattern formation in unstable thin liquid films," Physical Review Letters, vol. 81, no. 16, pp. 34633466, 1998.

[5] J. Rysz, "Monte Carlo simulations of phase separation in thin polymer blend films: scaling properties of morphological measures," Polymer, vol. 46, no. 3, pp. 977-982, 2005.

[6] A. M. Higgins and R. A. L. Jones, "Anisotropic spinodal dewetting as a mute to self-assembly of patterned surfaces," Nature, vol. 404, no. 6777, pp. 476-478, 2000.

[7] S. Puri and K. Binder, "Surface-directed spinodal decomposition: phenomenology and numerical results," Physical Review A, vol. 46, no. 8, pp. R4487-R4489, 1992.

[8] "nano-structured surfaces," August 2002, http://www.iop.org/.

[9] E. Klarreich, "Biologists join the dots," Nature, vol. 413, no. 6855, pp. 450-452, 2001.

[10] D. H. Cobden, "Molecular electronics: nanowires begin to shine," Nature, vol. 409, no. 6816, pp. 32-33, 2001.

[11] R. W. Cahn, P. Haasen, and E. J. Kramer, "Processing of ceramics," in Materials Science and Technology, vol. 17B, VCH, New York, NY, USA, 1994.

[12] P. Weiss, "To bead or not to bead? That is the question when researchers make ultrathin coatings," Science News, vol. 155, no. 2, pp. 28-30, 1999.

[13] K. Vijaya Sarathy and K. S. Narayan, "Novel properties exhibited by films of gold nanoparticle-polythiophene blends," Current Science, vol. 77, no. 5, pp. 678-681, 1999.

[14] R. Blossey, "Self-cleaning surfaces-virtual realities," Nature Materials, vol. 2, pp. 301-306, 2003.

[15] W. Zhao, M. H. Rafailovich, J. Sokolov et al., "Wetting properties of thin liquid polyethylene propylene films," Physical Review Letters, vol. 70, no. 10, pp. 1453-1456, 1993.

[16] J. Bischof, D. Scherer, S. Herminghaus, and P. Leiderer, "Dewetting modes of thin metallic films: nucleation of holes and spinodal dewetting," Physical Review Letters, vol. 77, no. 8, pp. 1536-1539, 1996.

[17] G. Reiter, "Dewetting of thin polymer films," Physical Review Letters, vol. 68, no. 1, pp. 75-78, 1992.

[18] R. Khanna, A. T. Jameel, and A. Sharma, "Stability and breakup of thin polar films on coated substrates: relationship to macroscopic parameters of wetting," Industrial and Engineering Chemistry Research, vol. 35, no. 9, pp. 3081-3092, 1996.

[19] S. P. Singh and M. Roychoudhury, "Dynamic Monte Carlo simulation for dewetting of thin polymer films on a repulsive substrate," Acta Ciencia Indica, vol. 33, pp. 571-578, 2007. 
[20] S. P. Singh and M. Roychoudhury, "Evolution of patterns in an unstable thin polymer film adsorbed on a solid substrate: a dynamic Monte Carlo study," Acta Ciencia Indica, vol. 34, pp. 61-70, 2008.

[21] R. Xie, A. Karim, J. F. Douglas, C. C. Han, and R. A. Weiss, "Spinodal dewetting of thin polymer films," Physical Review Letters, vol. 81, no. 6, pp. 1251-1254, 1998.

[22] M. Sferrazza, M. Heppenstall-Butler, R. Cubitt, D. Bucknall, J. Webster, and R. A. L. Jones, "Interfacial instability driven by dispersive forces: the early stages of spinodal dewetting of a thin polymer film on a polymer substrate," Physical Review Letters, vol. 81, no. 23, pp. 5173-5176, 1998.

[23] N. Goldenfeld, in Lectures on Phase Transitions and the Renormalization Group, Frontiers in Physics, Westview Press, 1992.

[24] L. D. Landau and E. M. Lifshitz, in Statistical Physics part 1, ' Nucleation in Phase transitions', Butterworth-Heinemann, 3rd edition, 1980.

[25] C. Redon, F. Brochard-Wyart, and F. Rondelez, "Dynamics of dewetting," Physical Review Letters, vol. 66, no. 6, pp. 715-718, 1991.

[26] A. Sharma and G. Reiter, "Instability of thin polymer films on coated substrates: rupture, dewetting, and drop formation," Journal of Colloid and Interface Science, vol. 178, no. 2, pp. 383399, 1996.

[27] S. P. Singh, "Dewetting: A pursuit (some technological aspects)," in Proceedings of the National Symposium on Advances in Material Science, p. 61, Department of Physics, DDU University Gorakhpur, March 2005.

[28] A. T. Jameel and A. Sharma, "Morphological phase separation in thin liquid films. II: equilibrium contact angles of nanodrops coexisting with thin films," Journal of Colloid And Interface Science, vol. 164, no. 2, pp. 416-427, 1994.

[29] A. Sharma, "Stability and breakup of thin evaporating water films: role of hydrophobic interaction," Journal of Colloid and Interface Science, vol. 199, no. 2, pp. 212-214, 1998.

[30] N. Samid-Merzel, S. G. Lipson, and D. S. Tannhauser, "Pattern formation in drying water films," Physical Review E, vol. 57, no. 3, pp. 2906-2913, 1998.

[31] P. Atkins and J. de Paula, "Hydrophobic interaction," in Physical Chemistry, Oxford University Press, Oxford, UK, 1995.

[32] G. Reiter, J. Schultz, P. Auroy, and L. Auvray, "Improving adhesion via connector polymers to stabilize non-wetting liquid films," Europhysics Letters, vol. 33, no. 1, pp. 29-34, 1996.

[33] R. R. Netz and D. Andelman, "Roughness-induced wetting," Physical Review E, vol. 55, no. 1, pp. 687-700, 1997.

[34] G. Reiter, "The artistic side of intermolecular forces," Science, vol. 282, no. 5390, pp. 888-889, 1998.

[35] B. D. Ermi, G. Nisato, J. F. Douglas, J. A. Rogers, and A. Karim, "Coupling between phase separation and surface deformation modes in self-organizing polymer blend films," Physical Review Letters, vol. 81, no. 18, pp. 3900-3903, 1998.

[36] V. S. Mitlin and N. V. Petviashvili, "Nonlinear dynamics of dewetting: kinetically stable structures," Physics Letters A, vol. 192, no. 5-6, pp. 323-326, 1994.

[37] K. Binder and D. W. Heermann, Monte Carlo Simulation in Statistical Physics, Springer, Heidelberg, Germany, 2nd edition, 2002.

[38] N. Goldenfeld, Lectures on Phase Transitions and the Renormalization Group, Frontiers in Physics, Westview Press, 1992.
[39] A. Milchev and K. Binder, "Static and dynamic properties of adsorbed chains at surfaces: Monte Carlo simulation of a bead-spring model," Macromolecules, vol. 29, no. 1, pp. 343354, 1996.

[40] R. Seemann, S. Herminghaus, C. Neto et al., "Dynamics and structure formation in thin polymer melt films," Journal of Physics Condensed Matter, vol. 17, no. 9, pp. S267-S290, 2005.

[41] V. R. Gwarikar, Vishwanathan, and J. Sreedhar, Polymer Science, New Age International Pvt Ltd., 2001.

[42] W. H. Press, S. A. Teukolsky, W. T. Vellerling, and B. P. Flannery, "Linear correlations," in Numerical Recipes, Cambridge University Press, New Delhi, India, 2nd edition, 1998.

[43] H. Margenau, "The role of quadrupole forces in van der Waals attractions," Physical Review, vol. 38, no. 4, pp. 747-756, 1931.

[44] S. P. Singh, "Some comments on the new approaches to experimental techniques for creation/assembling and investigation of micro/nanoscale confined system," in Proceedings of the National Conference on Experimental Tools for Material Science Research: State of the Art, MMV, BHU, India, Decemebr 2010. 

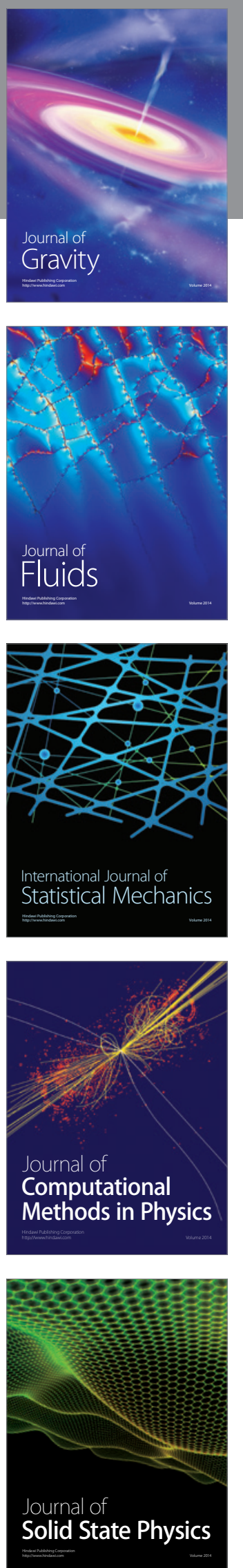

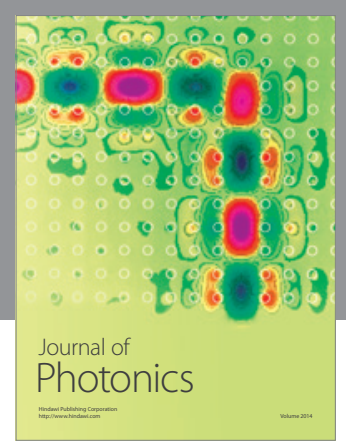

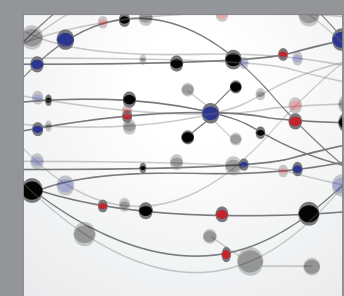

The Scientific World Journal
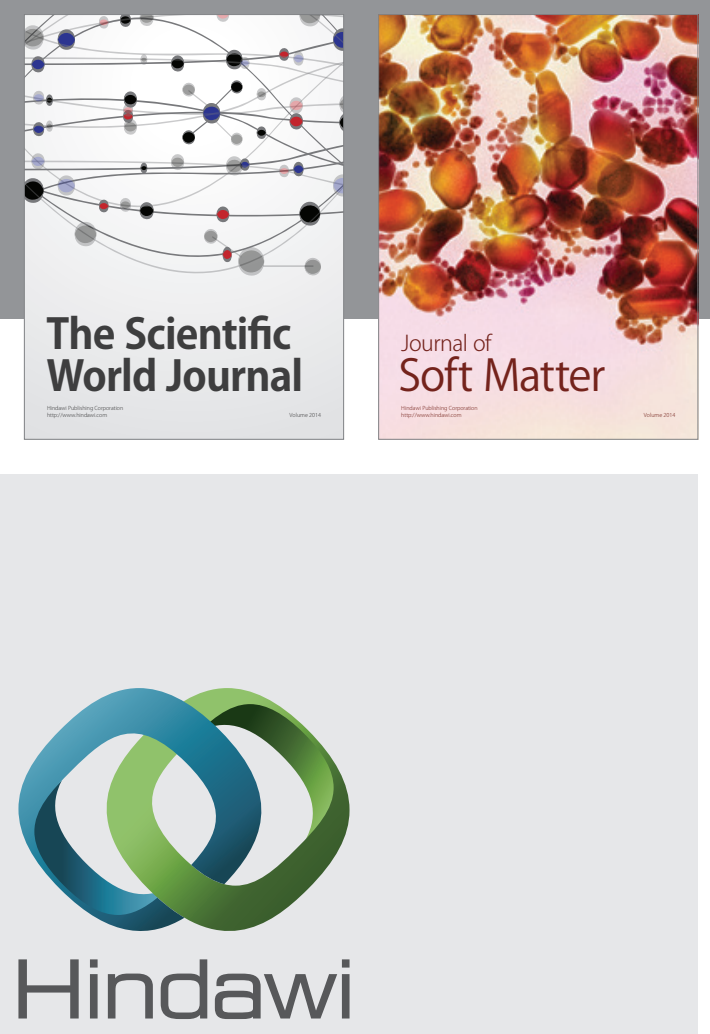

Submit your manuscripts at

http://www.hindawi.com
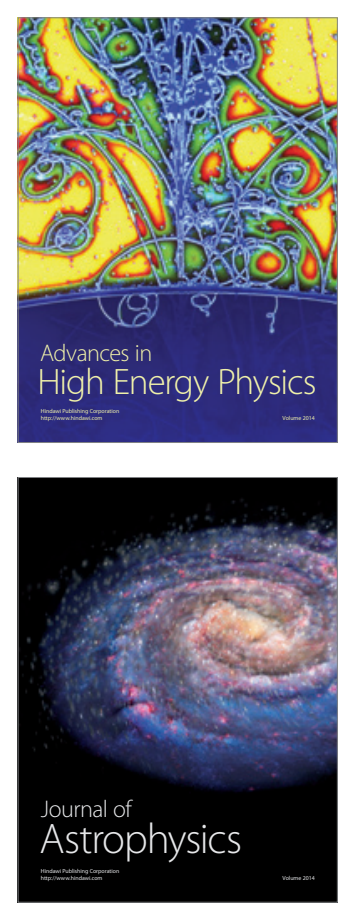
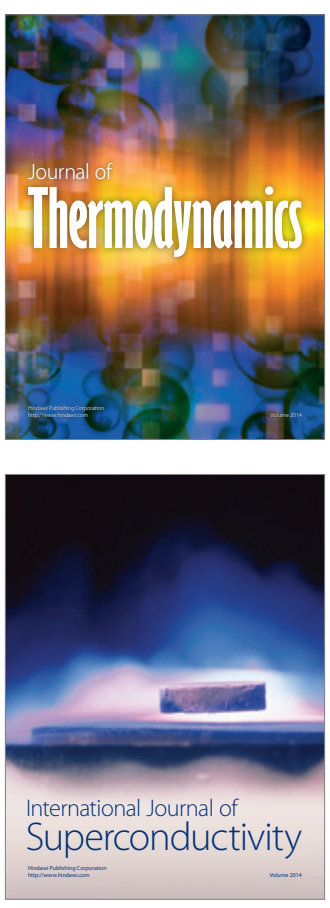
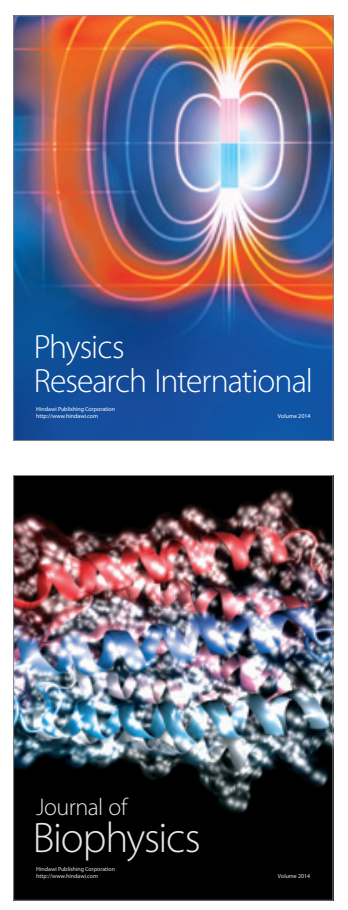
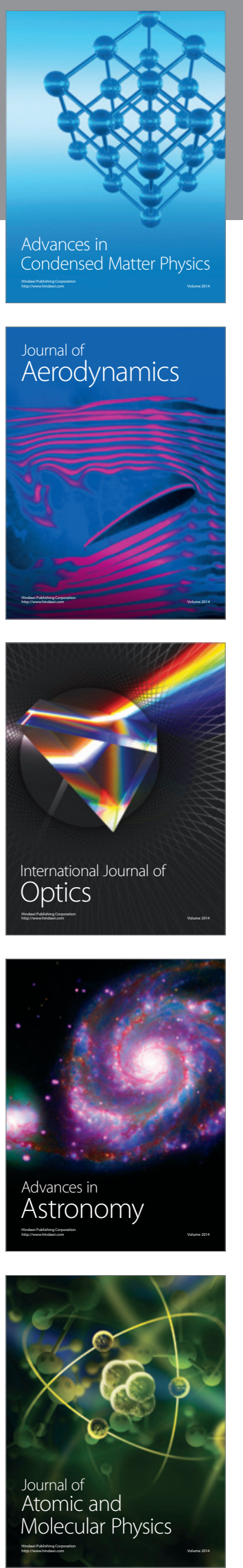